\title{
Novel alternative splicing isoform biomarkers identification from high-throughput plasma proteomics profiling of breast cancer
}

\author{
Fan Zhang ${ }^{1,2^{*}}$, Mu Wang ${ }^{3,4}$, Tran Michael ${ }^{1}$, Renee Drabier ${ }^{*}$ \\ From The International Conference on Intelligent Biology and Medicine (ICIBM 2013) \\ Nashville, TN, USA. 11-13 August 2013
}

\begin{abstract}
Background: In the biopharmaceutical industry, biomarkers define molecular taxonomies of patients and diseases and serve as surrogate endpoints in early-phase drug trials. Molecular biomarkers can be much more sensitive than traditional lab tests. Discriminating disease biomarkers by traditional method such as DNA microarray has proved challenging. Alternative splicing isoform represents a new class of diagnostic biomarkers. Recent scientific evidence is demonstrating that the differentiation and quantification of individual alternative splicing isoforms could improve insights into disease diagnosis and management. Identifying and characterizing alternative splicing isoforms are essential to the study of molecular mechanisms and early detection of complex diseases such as breast cancer. However, there are limitations with traditional methods used for alternative splicing isoform determination such as transcriptome-level, low level of coverage and poor focus on alternative splicing.

Results: Therefore, we presented a peptidomics approach to searching novel alternative splicing isoforms in clinical proteomics. Our results showed that the approach has significant potential in enabling discovery of new types of high-quality alternative splicing isoform biomarkers.

Conclusions: We developed a peptidomics approach for the proteomics community to analyze, identify, and characterize alternative splicing isoforms from MS-based proteomics experiments with more coverage and exclusive focus on alternative splicing. The approach can help generate novel hypotheses on molecular risk factors and molecular mechanisms of cancer in early stage, leading to identification of potentially highly specific alternative splicing isoform biomarkers for early detection of cancer.
\end{abstract}

\section{Introduction}

A biomarker as defined by the National Cancer Institute is "a biological molecule found in blood, other body fluids, or tissues that is a sign of a normal or abnormal process, or of a condition or disease [1]." It is a characteristic that is objectively measured and evaluated as an indicator of normal biological processes, pathogenic processes, or pharmacologic responses to a therapeutic intervention [2]. The field of biomarkers has grown extensively over the past decade in many areas such as

\footnotetext{
* Correspondence: Fan.Zhang@unthsc.edu; Renee.Drabier@unthsc.edu 'Department of Academic and Institutional Resources and Technology, University of North Texas Health Science Center, Fort Worth, USA Full list of author information is available at the end of the article
}

medicine, cell biology, genetics, geology and astrobiology, and ecotoxicology etc. and biomarkers are currently being studied in many academic centers and in industry.

In recent years, functional genomics studies using DNA Microarrays have been shown effective in identifying markers differentiating between breast cancer tissues and normal tissues, by measuring thousands of differentially expressed genes simultaneously [3-5].

However, early detection and treatment of breast cancer is still challenging. One reason is that obtaining tissue samples for microarray analysis can be still difficult. Another reason is that genes are not directly involved in any physical functions. On the contrary, the proteome, are the real functional molecules and the keys to 
understanding the development of cancer. Moreover, the fact that breast cancer is not a single homogeneous disease but consists of multiple disease status, each arising from a distinct molecular mechanism and having a distinct clinical progression path [6] makes the disease difficult to early detect.

Alternative splicing isoforms represent a new class of diagnostic biomarkers [7]. The chance of success with alternative splicing isoforms would be higher than the conventional approach $[8,9]$. Alternative splicing occurs in $95 \%$ human genes and works by selecting specific exons and sometimes even intronic regions of the gene into mature mRNAs [10]. Alternative splicing accounts for approximately $8 \%$ of all protein isoforms which is any of several different forms of the same protein and have three types: alternative splicing, SNP, and posttranslational modification (PTM).

Recent scientific studies have shown that diseased cells may produce many types of splicing variants of common regulatory proteins, e.g., protein kinase C, 14-3-3, p53, and VGFR, which could provide novel insights into complex disease diagnosis and management, particularly in cancers [11-14]. Alternative mRNA splicing is an important source of achieving molecular functional diversity. It is often regulated in a temporal or tissuespecific fashion, giving rise to different protein isoforms in different tissues or developmental states mediated by extracellular signaling mechanisms $[15,16]$. Splicing regulation is a key mechanism to tune gene expression to a variety of conditions and its dysfunction may often be at the basis of the onset of genetic disease and cancer [9]. In cancer, many examples of alternative splicing isoforms were reported [17-21]. For example, Julian et al. used a high-throughput reverse transcription-PCR-based system for splicing annotation to monitor the alternative splicing profiles of 600 cancer-associated genes in a panel of 21 normal and 26 cancerous breast tissues. They found that 41 alternative splicing events significantly differed in breast tumors relative to normal breast tissues and that most cancer-specific changes in splicing that disrupt known protein domains support an increase in cell proliferation or survival consistent with a functional role for alternative splicing in cancer. Compared to normal mRNA splicing events, alternative splicing mechanisms and patterns in complex diseases such as cancer can be quite complex. Finding alternative splicing isoforms or patterns of their development, therefore, have been promising in helping develop high-quality biomarkers and targets for disease management [22].

Discovering disease biomarkers in the human plasma has been met with both enthusiasm and criticisms in recent years. On one hand, it is expected that disease conditions such as cancer may be diagnosed early by analyzing complex protein mixtures in easily accessible human blood (serum or plasma), in which proteins induced by cancer may be differentially cleaved, secreted, leaked out, and therefore differentially detected from normal healthy conditions. On the other hand, the bulk of proteins circulating in human blood vary in different conditions without cancers and across different individuals, and changes in protein expressions are often diluted in the blood - extremely challenging for biological interpretation of protein quantification changes $[23,24]$. The use of alternative splicing isoforms as potential biomarkers, therefore, offers a new opportunity to use the detectability instead of quantification of biomarker peptides, for the peptides that may map to unique alternative splicing isoforms specific to cancer.

However, systematic, comprehensive, proteome-scale experimental and computational characterization of protein isoforms directly at the protein/peptide level and with exclusive focus on alternative splicing has never been reported. Compared with the "indirect" transcriptome-level characterizations such as EST sequencing [25], exon array[26], exon-exon junction array[27], and next-generation sequencing of all mRNA transcripts $[28,29]$, "direct" proteome-level characterization of alternative splicing isoforms can address the wide-spread concern that mRNA expressions and protein expression do not correlate well in a clinical proteomics [30,31]. In 2010, we developed the PEPtidomics Protein Isoform Database (PEPPI [32], http://bio.informatics.iupui.edu/ peppi), a database of computationally-synthesized human peptides that can identify protein isoforms derived from either alternatively spliced mRNA transcripts or SNP variations. Although the PEPPI database is the first peptidomics protein isoform database, it is not exclusive to alternative splicing and has poor coverage of alternative splicing.

Therefore, based on the PEPPI [32], we presented a new peptidomics approach to searching novel alternative splicing isoform in proteomics data and demonstrated that the approach can help researchers identify and characterize novel alternative splicing isoform from experimental proteomics data and discover diagnostic value and clinical significance.

\section{Materials and methods}

\section{Human plasma samples}

Plasma protein profiles were collected by the Hoosier Oncology Group (HOG) (Indianapolis, IN, USA). Each sample was analyzed in a single batch by mass spectrometry. In the study, 80 plasma samples were collected (40 samples collected from women with breast cancer and 40 from healthy volunteer woman who served as controls). An independent validation dataset of 80 samples which contains 40 samples collected from women diagnosed with breast cancer and 40 from healthy 
volunteer woman who served as controls were collected by the Hoosier Oncology Group (HOG) (Indianapolis, IN, USA) too and is comparable to the study in the demography and clinical distribution of breast cancer stages/subtypes. For example, most of patients involved in the two studies were diagnosed with an early stage breast cancer (stage I or II), fell into age group between 40 and 65 , and had mean tumor size of 2.2 .

\section{Protein identification and quantification}

For protein identification, Tryptic peptides were analyzed using Thermo-Fisher Scientific linear ion-trap mass spectrometer (LTQ) coupled with a Surveyor HPLC system. Peptides were eluted with a gradient from 5 to $45 \%$ Acetonitrile developed over 120 minutes and data were collected in the triple-play mode (MS Scan, zoom scan, and MS/MS scan). The acquired raw peak list data were generated by XCalibur (version 2.0) using default parameters and further analyzed by the label-free identification and quantitative algorithm using default parameters described by Higgs et al [33]. MS database searches were performed against the combined protein data set from International Protein Index and the non-redundant NCBI-nr human protein database, which totaled 22,180 protein records. Carious data processing filters for protein identification were applied to keep only peptides with the XCorr score above 1.5 for singly charged peptides, 2.5 for doubly charged peptides, and 3.5 for triply charged peptides. These XCorr scores were set according to linear discriminant analysis similar to that described in DTASelect (version 2.0) to control false-positive rate at below $5 \%$ levels.

For protein quantification, first, all extracted ion chromatograms (XICs) were aligned by retention time. Each aligned peak were matched by precursor ion, charge state, fragment ions from MS/MS data, and retention time within a one-minute window. Then, after alignment, the area-under-the-curve (AUC) for each individually aligned peak from each sample was measured, normalized, and compared for relative abundance-all as described in [33]. Here, a linear mixed model generalized from individual ANOVA (Analysis of Variance) was used to quantify protein intensities. In principle, the linear mixed model considers three types of effects when deriving protein intensities based on weighted average of quantile-normalized peptide intensities: 1) group effect, which refers to the fixed non-random effects caused by the experimental conditions or treatments that are being compared; 2) sample effect, which refers to the random effects (including those arising from sample preparations) from individual biological samples within a group; 3$)$ replicate effect, which refers to the random effects from replicate injections from the same sample preparation.

\section{Peptidomics approach to searching novel alternative splicing isoform in proteomics data}

Our peptidomics approach to identifying novel alternative splicing isoform in proteomics data includes three steps (Figure 1): 1) building an synthetic database of

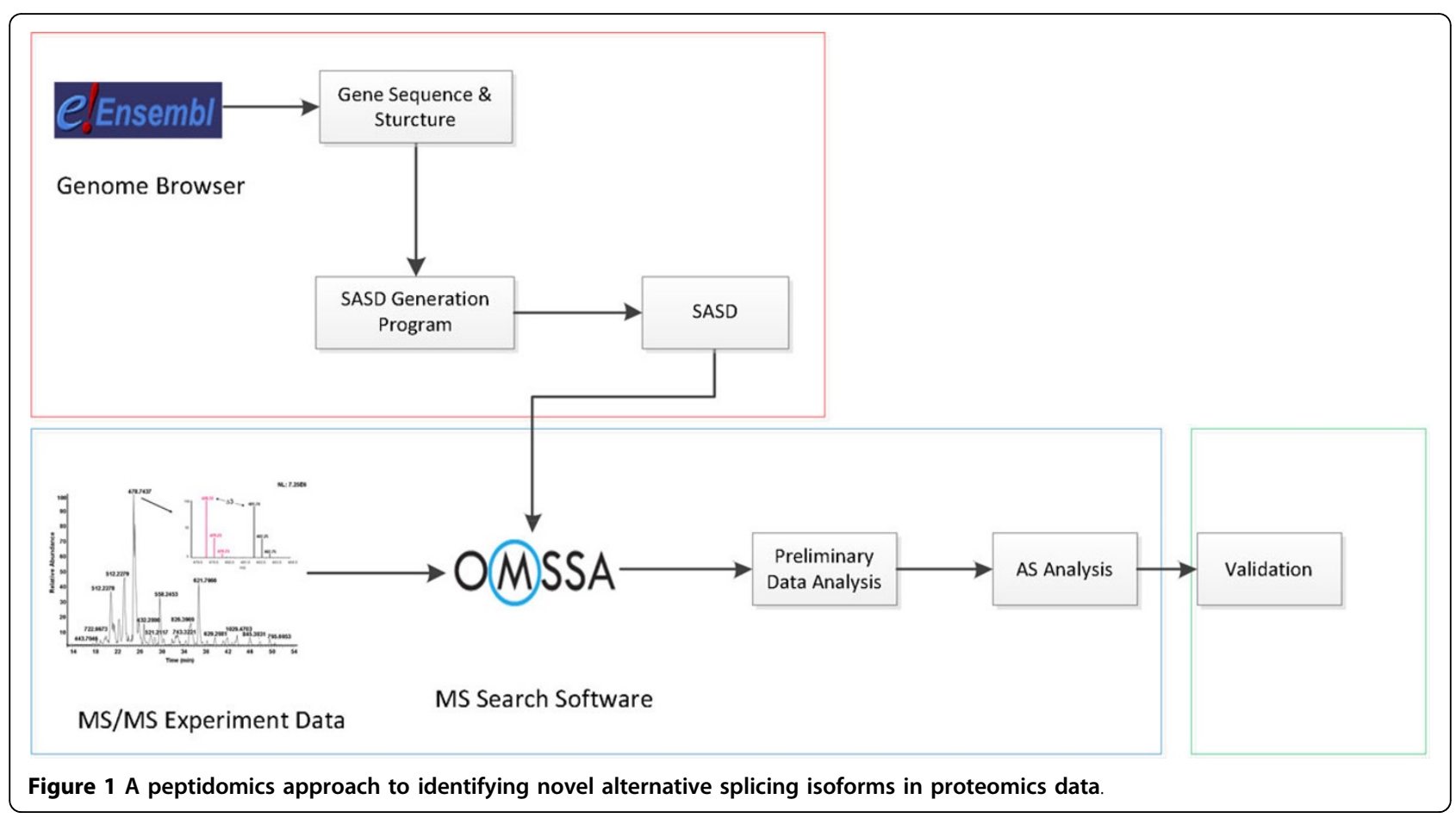


alternative splicing isoforms for proteomics experiments; 2) identification, characterization of alternative splicing isoform using proteomics; and 3) validation of alternative splicing isoform.

\section{Step 1: building a synthetic database of alternative splicing isoforms for proteomics experiments}

Briefly, we developed the Synthetic Alternative Splicing Database (SASD) [34] in three steps: 1)extracting information on gene structures of all genes in the human genome and incorporating the IPAD database [35], 2) compiling artificial splicing transcripts, and 3) translating the artificial transcripts into alternative splicing peptides.

In the first step, we use the BioMart to extract information on all human genes in the Ensemble [36] from the Homo sapiens genes dataset (GRCh37.p10) in the Ensembl Genes 71 database. We then extract information on each human gene's position, name, exon/intron coordinates, exon phase, sequences, and annotation.

In the second step, we generate artificial splicing transcript (AST), which is an exhaustive compilation of two categories of peptides. The first category is the peptides translated from all single exons and introns, and the second category is the peptides that cover all theoretically possible exon/intron junction regions of all genes in the human genome. The first category contains two types of alternative splicing: single exon(EXON_NM) and single intron (INTRON_AS). The second category contains four types of alternative splicing: intron-exon (I_E_AS, left intron retention junction), exon-intron (E_I_AS, right intron retention junction), neighboring exon-exon (E_E_NM, normal splicing junction) and non-neighboring exon-exon (E_E_AS, exon skipping junction).

In the third step, we directly use the phase to translate the sequence for the exons with the phase information in Ensemble transcript. For the exons without the phase information in Ensemble transcript, three open reading frames are used in translating into three peptides and the longest peptide crossing the splicing site is reserved as alternative splicing peptide for SASD.

\section{Step 2: identification and characterization of alternative splicing isoform using proteomics}

The most popular three types of search algorithms are 1) correlating acquired MS/MS with theoretical spectrum, counts the number of peaks in common, such as: SEQUEST [37] and X!Tandem [38], 2) modeling the extent of peptide fragmentation, then estimates the probability that an assignment is incorrect due specifically to a random match, such as Mascot [39] and OMSSA [40], and 3) De Novo Sequencing such as Lutefisk[41] and PEAKS[42]. We first run OMSSA against
SASD to identify peptide from MS data. Then we perform preliminary data analysis. Last, we extract information about alternative splicing.

OMSSA reports hits ranked by E-value. An E-value for a hit is a score that is the expected number of random hits from a search library to a given spectrum, such that the random hits have an equal or better score than the hit. For example, a hit with an E-value of 1.0 implies that one hit with a score equal to or better than the hit being scored would be expected at random from a sequence library search [40]. The E-value is calculated to report the expected frequency of observing scores equivalent to or better than the one for the reported peptide if the results were to take place randomly. The lower the E-value is, the more significant the score for the identified peptide by the peptide search using SASD database is.

One-sided Wilcoxon signed-rank test is used to perform the preliminary statistical analysis in order to identify peptides with significant occurrence differences in the health and breast cancer samples.

\section{Step 3: validation of alternative splicing isoform}

We present two kinds of methods to validate isoforms in proteomics data, which are 1) literature curation of alternative splicing isoforms and 2) cross-validation of multiple studies. First, we perform an extensive literature curation to determine the constituents of alternative splicing isoform. Then, we validate results using independent proteomics datasets derived from other study. We believe that such an integrative systems approach is essential to development and validation of panel alternative splicing isoform that may withstand rigorous testing for the future steps.

\section{Pathway analysis}

Pathway analysis is performed using the following databases: Integrated Pathway Analysis Database (IPAD) (http://bioinfo.hsc.unt.edu/ipad/) [43].

\section{Results}

The 80 breast cancer plasma samples with 40 samples from women diagnosed with breast cancer and 40 from healthy volunteer women as controls were searched by OMSSA[40] against the SASD database. After OMSSA searching, preliminary statistical analysis, and alternative splicing, we identified the eight alternative splicing isoform biomarkers using the peptidomics approach to searching novel alternative splicing isoform in the proteomics data (Table 1). The peptides with E-value greater than 0.01 were filtered out. The P-value was calculated by performing one-sided Wilcoxon signed-rank test to examine the probability that the median difference between two groups of samples is greater than zero. The number of such peptide found in Health (h) and Cancer 
Table 1 Novel alternative splicing isoform candidate biomarkers for breast cancer in plasma

\begin{tabular}{|c|c|c|c|c|c|c|c|c|}
\hline Peptide sequence & gene & transcript & mode & type & pvalue & $\mathrm{h}$ & c & Peptide \\
\hline & & & & & & & & Atlas \\
\hline SWGGRPQRMGAVPGGWWSAVLMGGAR & ERBB2 & ENST00000269571 & ¡18 & INTRON_AS & 9.48E-05 & 4 & 20 & No \\
\hline QTPKHISESLGAEVDPDMSWSSSLATPPTLSSTVLI(G)LLHSSVK & BRCA2 & ENST00000380152 & E7_E11 & E_E_AS & 8.57E-04 & 1 & 12 & No \\
\hline SLWLQSQPHFCCFWLTVTFPPPLQ^THRELAQSSHAQR & NTRK3 & ENST00000317501 & i2_E3 & I_E_AS & $1.22 \mathrm{E}-02$ & 2 & 10 & No \\
\hline WGLLLALLPPGAASTQ(A)VWTWMTR & ERBB2 & ENST00000269571 & E1_E16 & E_E_AS & $1.22 \mathrm{E}-02$ & 2 & 10 & No \\
\hline LSWNHVARALTLTQSLVSSVTSGK & NTRK3 & ENST00000559764 & $\mathrm{i} 2$ & INTRON_AS & 1.39E-02 & 4 & 13 & No \\
\hline $\mathbf{C Q}(\mathrm{G})$ EPYHDIRFNLMAWPDR & BAP1 & ENST00000460680 & E3_E9 & E_E_AS & 3.33E-02 & 9 & 18 & No \\
\hline QVLP^VGVLGPPGQQAPPPYPGPHPAGPPVIQQPTTPMFVAPPPK & PBRM1 & ENST00000296302 & E9_E29 & E_E_AS & 3.89E-02 & 6 & 14 & No \\
\hline DHLACW^DYDLCITCYNTKNHDHK & EP300 & ENST00000263253 & E22_E31 & E_E_AS & 4.50E-02 & 4 & 11 & No \\
\hline
\end{tabular}

(c) samples are listed separately in the table. The tested peptides are more likely to exist in cancer samples than in healthy samples when P-value is small. Bold text is the left part of the junction and italic text is the right part. Splicing site is marked by ${ }^{\wedge}$ or (). '()' means the splicing site is shared by the left region and right region. For example, the second peptide QTPKHISESLGAEVDP DMSWSSSLATPPTLSSTVLI(G)LLHSSVK is a synthetic product of the ENST00000380152 in gene BRCA2 when its eighth, ninth, and tenth exons are skipped and its seventh exon is combined together with its eleventh exon. The Glycine is the shared splicing site between the seventh exon and the eleventh exon.

None of the eight peptides are reported to have ever been detected in the Peptide Atlas database, which contains a comprehensive catalogue of all peptides derived from published proteomics experiments. They are not found with normal splicing mechanism (Table 1).

The first sequence is a single intron alternative splicing. It was observed in 20 patient samples and 4 healthy samples. Triple play mode of the annotated Thermo-Finnegan LCQ-DECA ion-trap MS/MS spectrum is shown in Figure 2[44]. The triple play mode includes a) primary mass spectrum; b) zoom scan mass spectrum; c) MS/MS mass spectrum and d) protein identification from MS/MS). Due to space limit, the spectrums of other seven alternative splicing sequences are omitted.

The eight peptides, identified by OMSSA, have significant difference in the numbers of hit samples between healthy women and breast cancers (pvalue $<0.05$, Table 1). A screen shot from the UCSC genome browser [45] in the region of these peptides are also shown in Figure 3 . It shows that these peptide sequences are not found in EST sequences and mRNA from Genbank and one refseq gene(Figure 3).

Table 1 shows that our peptidomics approach has significant potential in enabling discovery of new types of high-quality alternative splicing isoform biomarkers. Further literature search found that there are no any literature reports for the eight peptides. Moreover, a cross-validation found that identification of the eight peptides is supported by the independent Study which contains 40 samples collected from women diagnosed with breast cancer and 40 from healthy volunteer woman who served as controls.

Pathway analysis shows the pathways linked with the eight alternative splicing isoforms are transcription factor, signaling, cancer, and synthesis (Table 2).

\section{Discussions}

We described the peptidomics approach to searching novel alternative splicing isoform in proteomics data, especially artificial alternative splicing and SNP. We can use it to identify two types of common alternative splicing events: Exon Skipping and Intron Retention. Exon Skipping is an alternative splicing mechanism in which exon(s) are included or excluded from the final gene transcript leading to extended or shortened mRNA variants. And Intron Retention is an event in which an intron is retained in the final transcript. Other types of alternative splicing events such as alternative 3' splice site and $5^{\prime}$ splice site are not included in our method but can be derived indirectly from the two basic types: exon skipping and intron retention.

The current protein sequence databases used by tandem mass spectra search engines, for example IPI, UniProt, and NCBI nr, are designed to be useful as possible to as many researchers as possible. As such, they are a less than ideal substrate for tandem mass spectra search. Protein sequence databases typically represent only "full-length" protein sequences and attempt to collapse protein variants to a single "consensus" entry. Tandem mass spectra search engines, however, chop up the protein sequence using an in-silico enzymatic digestion (such as trypsin), so full-length proteins are not needed in order to identify experimentally observed peptides; and the currently available search engines require the experimental peptides' sequences be explicitly present in the sequence database in order to identify them, so explicit sequence variants are very important. The SASD is in fact a complete peptide sequence database, which 

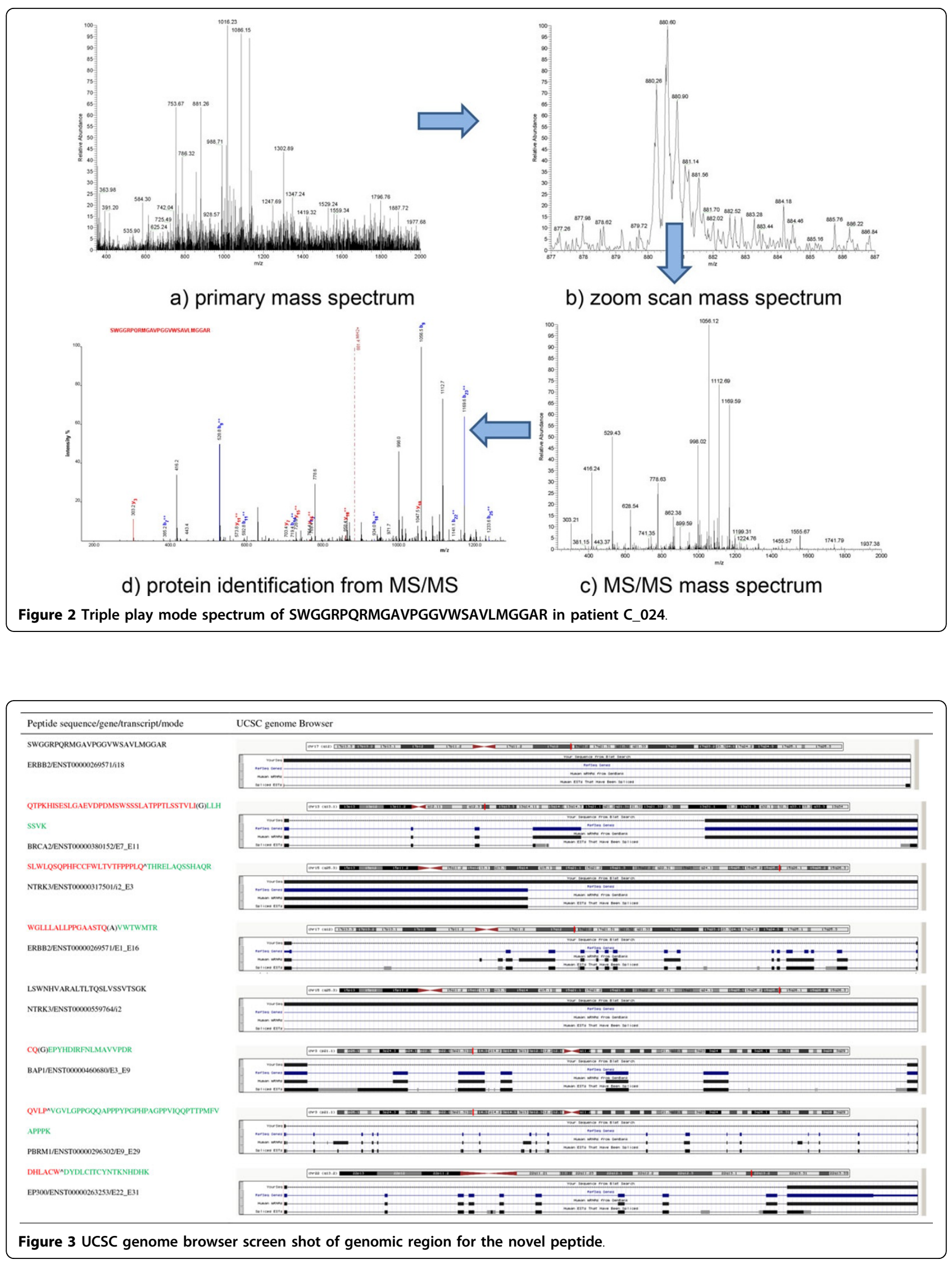
Table 2 Pathway analysis for the eight alternative splicing isoforms.

\begin{tabular}{|c|c|c|}
\hline PathwayID & PathwayName & Molecule \\
\hline 200017 & p73 transcription factor network & BRCA2;EP300 \\
\hline 200141 & FOXM1 transcription factor network & EP300;BRCA2 \\
\hline 200200 & Validated targets of C-MYC transcriptional repression & EP300;ERBB2 \\
\hline hsa04520 & Adherens junction & ERBB2;EP300 \\
\hline hsa05212 & Pancreatic cancer & ERBB2;BRCA2 \\
\hline 1306955 & GRB7 events in ERBB2 signaling & ERBB2 \\
\hline 1358803 & Downregulation of ERRB2:ERBB3 signaling & ERBB2 \\
\hline 168253 & Host Interactions with Influenza Factors & PBRM1 \\
\hline 168268 & Virus Assembly and Release & PBRM1 \\
\hline 168270 & Fusion and Uncoating of the Influenza Virion & PBRM1 \\
\hline 168275 & Entry of Influenza Virion into Host Cell via Endocytosis & PBRM1 \\
\hline 168303 & Packaging of Eight RNA Segments & PBRM1 \\
\hline 168330 & Viral RNP Complexes in the Host Cell Nucleus & PBRM1 \\
\hline 192814 & vRNA Synthesis & PBRM1 \\
\hline 192869 & cRNA Synthesis & PBRM1 \\
\hline 200159 & ErbB receptor signaling network & ERBB2 \\
\hline 168277 & Influenza Virus Induced Apoptosis & PBRM1 \\
\hline 168288 & Fusion of the Influenza Virion to the Host Cell Endosome & PBRM1 \\
\hline 168298 & Release & PBRM1 \\
\hline 168302 & Budding & PBRM1 \\
\hline 168336 & Uncoating of the Influenza Virion & PBRM1 \\
\hline 192905 & vRNP Assembly & PBRM1 \\
\hline 73951 & Homologous recombination repair of replication-independent double-strand breaks & BRCA2 \\
\hline 918233 & TRAF3-dependent IRF activation pathway & EP300 \\
\hline 73888 & Homologous Recombination Repair & BRCA2 \\
\hline 76003 & Presynaptic phase of homologous DNA pairing and strand exchange & BRCA2 \\
\hline 76010 & Homologous DNA pairing and strand exchange & BRCA2 \\
\hline h_hifPathway & Hypoxia-Inducible Factor in the Cardiovascular System & EP300 \\
\hline h_pitx2Pathway & Multi-step Regulation of Transcription by Pitx2 & EP300 \\
\hline h_ppargPathway & Role of PPAR-gamma Coactivators in Obesity and Thermogenesis & EP300 \\
\hline h_RELAPathway & Acetylation and Deacetylation of RelA in The Nucleus & EP300 \\
\hline hsa05200 & Pathways in cancer & ERBB2;BRCA2;EP300 \\
\hline h_carm1Pathway & Transcription Regulation by Methyltransferase of CARM1 & EP300 \\
\hline h_melanocytepathway & Melanocyte Development and Pigmentation Pathway & EP300 \\
\hline h_pelp1Pathway & Pelp1 Modulation of Estrogen Receptor Activity & EP300 \\
\hline h_vdrPathway & Control of Gene Expression by Vitamin D Receptor & EP300 \\
\hline hsa05215 & Prostate cancer & ERBB2;EP300 \\
\hline h_il7Pathway & IL-7 Signal Transduction & EP300 \\
\hline 168325 & Viral Messenger RNA Synthesis & PBRM1 \\
\hline 1963640 & GRB2 events in ERBB2 signaling & ERBB2 \\
\hline 419524 & Fanconi Anemia pathway & BRCA2 \\
\hline 73890 & Double-Strand Break Repair & BRCA2 \\
\hline 416572 & Sema4D induced cell migration and growth-cone collapse & ERBB2 \\
\hline h_g2Pathway & Cell Cycle: G2/M Checkpoint & EP300 \\
\hline h_mef2dPathway & Role of MEF2D in T-cell Apoptosis & EP300 \\
\hline h_atrbrcaPathway & Role of BRCA1, BRCA2 and ATR in Cancer Susceptibility & BRCA2 \\
\hline h_her2Pathway & Role of ERBB2 in Signal Transduction and Oncology & EP300 \\
\hline h_nthiPathway & NFkB activation by Nontypeable Hemophilus influenzae & EP300 \\
\hline h_p53hypoxiaPathway & Hypoxia and p53 in the Cardiovascular system & EP300 \\
\hline h_tgfbPathway & TGF beta signaling pathway & EP300 \\
\hline
\end{tabular}


Table 2 Pathway analysis for the eight alternative splicing isoforms. (Continued)

\begin{tabular}{lll}
\hline 1250196 & SHC1 events in ERBB2 signaling & ERBB2 \\
200025 & Signaling events mediated by HDAC Class III & EP300 \\
hsa03440 & Homologous recombination & BRCA2 \\
200164 & Retinoic acid receptors-mediated signaling & EP300 \\
400685 & Sema4D in semaphorin signaling & ERBB2 \\
933541 & TRAF6 mediated IRF7 activation & EP300 \\
\hline
\end{tabular}

includes majority of all occurrence of alternative splicing. It also provides alternative splicing for each peptide, such as splicing mode, splicing type, splicing site, starting position, ending position, and peptide sequence.

Moreover, the current protein sequence databases and some alternative splicing database such as ASTD and EID are not ideal or enough for identifying alternative splicing isoform from tandem mass spectrometry. There are either no or very few isoform information in these databases. For example, ASTD only includes 9757 occurrences of intron isoforms and 5214 occurrences of exon isoforms. Even for Cassette exons event, the number of occurrences is only $12470[15]$. In contrast, the SASD database in our method includes $11,919,779$ Alternative Splicing peptides covering about 56,630 genes (ensembl gene IDs), 95,260 transcripts (ensembl transcript IDs), 1956 pathways, 6704 diseases, 5615 drugs, and 52 organs.

Its comprehensive coverage means better sensitivity in identifying novel alternative splicing isoforms than the PEPPI. And its exclusive focus on alternative splicing can definitely increase the specificity of the identification of alternative splicing.

Alternative splicing isoform biomarkers are apparently important and can serve as an alternative to traditional biomarkers. We can use quantitative information such as $\mathrm{p}$ value to determine the significance of the marker. We can also use the qualitative information such as: splicing type, splicing mode, peptide sequence etc. to further analyze the alternative splicing's mechanism. We think that combination of traditional biomarkers with the alternative splicing isoform biomarkers will definitely help us better understand the treatment, diagnosis, and prognosis of cancer.

\section{Competing interests statement}

The authors declare that they have no competing interests.

\section{Authors' contributions}

$\mathrm{RD}$ and FZ conceived the initial work and designed the method. FZ and RD developed the alternative splicing database and method, and performed the computational analyses. MW provided experimental data. TM performed literature search. All authors are involved in the drafting and revisions of the manuscript.

\section{Acknowledgements}

We thank Hoosier Oncology Group for collecting breast cancer plasma samples. The proteomics study for biomarker discovery was supported by the National Cancer Institute Clinical Proteomics Technology Assessment for Cancer program (U24 CA126480). We also thank the support of Bioinformatics Program at University of North Texas Health Science Center.

\section{Declarations}

The publication costs for this article were funded by the bioinformatics program in University of North Texas Health Science Center.

This article has been published as part of BMC Systems Biology Volume 7 Supplement 5, 2013: Selected articles from the International Conference on Intelligent Biology and Medicine (ICIBM 2013): Systems Biology. The full contents of the supplement are available online at http://www. biomedcentral.com/bmcsystbiol/supplements/7/S5.

\section{Authors' details}

'Department of Academic and Institutional Resources and Technology, University of North Texas Health Science Center, Fort Worth, USA. ²Department of Forensic and Investigative Genetics, University of North Texas Health Science Center, Fort Worth, USA. ${ }^{3}$ Department of Biochemistry and Molecular Biology, IU School of Medicine, Indianapolis, IN 46202, USA. ${ }^{4}$ Indiana Center for Systems Biology and Personalized Medicine, Indianapolis, IN 46202, USA.

\section{Published: 9 December 2013}

\section{References}

1. National Cancer Institute Dictionary.

2. Biomarkers and surrogate endpoints: preferred definitions and conceptual framework. Clinical pharmacology and therapeutics 2001, 69(3):89-95.

3. Hu X, Zhang Y, Zhang A, Li Y, Zhu Z, Shao Z, Zeng R, Xu LX: Comparative serum proteome analysis of human lymph node negative/positive invasive ductal carcinoma of the breast and benign breast disease controls via label-free semiquantitative shotgun technology. OMICS 2009, 13(4):291-300.

4. Zeidan BA, Cutress RI, Murray N, Coulton GR, Hastie C, Packham G, Townsend PA: Proteomic analysis of archival breast cancer serum. Cancer Genomics Proteomics 2009, 6(3):141-147.

5. Lebrecht A, Boehm D, Schmidt M, Koelbl H, Schwirz RL, Grus FH: Diagnosis of breast cancer by tear proteomic pattern. Cancer Genomics Proteomics 2009, 6(3):177-182.

6. Polyak K: Breast cancer: origins and evolution. J Clin Invest 2007, 117(11):3155-3163

7. Roberts TK, Eugenin EA, Morgello S, Clements JE, Zink MC, Berman JW: $\mathrm{PrPC}$, the cellular isoform of the human prion protein, is a novel biomarker of HIV-associated neurocognitive impairment and mediates neuroinflammation. Am J Pathol 2010, 177(4):1848-1860.

8. Brinkman BM: Splice variants as cancer biomarkers. Clinical biochemistry 2004, 37(7):584-594.

9. Garcia-Blanco MA, Baraniak AP, Lasda EL: Alternative splicing in disease and therapy. Nat Biotechnol 2004, 22(5):535-546.

10. Kornblihtt AR, Schor IE, Allo M, Dujardin G, Petrillo E, Munoz MJ: Alternative splicing: a pivotal step between eukaryotic transcription and translation. Nature reviews Molecular cell biology 2013, 14(3):153-165.

11. Anensen N, Oyan AM, Bourdon JC, Kalland KH, Bruserud O, Gjertsen BT: A distinct $\mathrm{p} 53$ protein isoform signature reflects the onset of induction chemotherapy for acute myeloid leukemia. Clin Cancer Res 2006, 12(13):3985-3992.

12. Assender JW, Gee JM, Lewis I, Ellis IO, Robertson JF, Nicholson RI: Protein kinase $C$ isoform expression as a predictor of disease outcome on endocrine therapy in breast cancer. J Clin Pathol 2007, 60(11):1216-1221. 
13. Cressey R, Wattananupong $\mathrm{O}$, Lertprasertsuke $\mathrm{N}$, Vinitketkumnuen $\mathrm{U}$ : Alteration of protein expression pattern of vascular endothelial growth factor(VEGF) from soluble to cell-associated isoform during tumourigenesis. BMC Cancer 2005, 5:128.

14. Di Fede G, Giaccone G, Limido L, Mangieri M, Suardi S, Puoti G, Morbin M, Mazzoleni G, Ghetti B, Tagliavini F: The epsilon isoform of 14-3-3 protein is a component of the prion protein amyloid deposits of GerstmannStraussler-Scheinker disease. J Neuropathol Exp Neurol 2007, 66(2):124-130.

15. Thanaraj TA, Stamm S, Clark F, Riethoven JJ, Le Texier V, Muilu J: ASD: the Alternative Splicing Database. Nucleic Acids Res 2004, 32(Database):D64-69.

16. Matter $\mathrm{N}$, Herrlich $\mathrm{P}$, Konig $\mathrm{H}$ : Signal-dependent regulation of splicing via phosphorylation of Sam68. Nature 2002, 420(6916):691-695.

17. Lixia M, Zhijian C, Chao S, Chaojiang G, Congyi Z: Alternative splicing of breast cancer associated gene BRCA1 from breast cancer cell line. J Biochem Mol Biol 2007, 40(1):15-21.

18. Zhu Z, Xing S, Cheng P, Zeng F, Lu G: Modification of alternative splicing of Bcl-x pre-mRNA in bladder cancer cells. J Huazhong Univ Sci Technolog Med Sci 2006, 26(2):213-216.

19. $\mathrm{Ku} \mathrm{TH}, \mathrm{Hsu}$ FR: Mining colon cancer specific alternative splicing in EST database. AMIA Annu Symp Proc 2005, 1012.

20. Ogawa T, Shiga K, Hashimoto S, Kobayashi T, Horii A, Furukawa T: APAF-1ALT, a novel alternative splicing form of APAF-1, potentially causes impeded ability of undergoing DNA damage-induced apoptosis in the LNCaP human prostate cancer cell line. Biochem Biophys Res Commun 2003, 306(2):537-543.

21. Venables JP, Klinck R, Bramard A, Inkel L, Dufresne-Martin G, Koh C, GervaisBird J, Lapointe E, Froehlich U, Durand M, et al: Identification of alternative splicing markers for breast cancer. Cancer Res 2008, 68(22):9525-9531.

22. Skotheim Rl, Nees M: Alternative splicing in cancer: noise, functional, or systematic? Int J Biochem Cell Biol 2007, 39(7-8):1432-1449.

23. Rifai N, Gillette MA, Carr SA: Protein biomarker discovery and validation: the long and uncertain path to clinical utility. Nat Biotechnol 2006, 24(8):971-983.

24. Kulasingam V, Diamandis EP: Strategies for discovering novel cancer biomarkers through utilization of emerging technologies. Nature clinical practice Oncology 2008, 5(10):588-599.

25. Adams MD, Kelley JM, Gocayne JD, Dubnick M, Polymeropoulos MH, Xiao H, Merril CR, Wu A, Olde B, Moreno RF, et al: Complementary DNA sequencing: expressed sequence tags and human genome project. Science 1991, 252(5013):1651-1656.

26. Kapur K, Xing Y, Ouyang Z, Wong WH: Exon arrays provide accurate assessments of gene expression. Genome Biol 2007, 8(5):R82.

27. Johnson JM, Castle J, Garrett-Engele P, Kan Z, Loerch PM, Armour CD, Santos R, Schadt EE, Stoughton R, Shoemaker DD: Genome-wide survey of human alternative pre-mRNA splicing with exon junction microarrays. Science 2003, 302(5653):2141-2144.

28. Pan Q, Shai O, Lee LJ, Frey BJ, Blencowe BJ: Deep surveying of alternative splicing complexity in the human transcriptome by high-throughput sequencing. Nat Genet 2008, 40(12):1413-1415.

29. Pando MP, Kotraiah V, McGowan K, Bracco L, Einstein R: Alternative isoform discrimination by the next generation of expression profiling microarrays. Expert Opin Ther Targets 2006, 10(4):613-625.

30. Lee PS, Shaw LB, Choe LH, Mehra A, Hatzimanikatis V, Lee KH: Insights into the relation between mrna and protein expression patterns: II. Experimental observations in Escherichia coli. Biotechnol Bioeng 2003, 84(7):834-841.

31. Mehra A, Lee $\mathrm{KH}$, Hatzimanikatis $\mathrm{V}$ : Insights into the relation between mRNA and protein expression patterns: I. Theoretical considerations. Biotechnol Bioeng 2003, 84(7):822-833.

32. Zhou A, Zhang F, Chen JY: PEPPI: a peptidomic database of human protein isoforms for proteomics experiments. BMC bioinformatics 2010, , 11 Suppl 6: S7.

33. Higgs RE, Knierman MD, Gelfanova V, Butler JP, Hale JE: Comprehensive label-free method for the relative quantification of proteins from biological samples. J Proteome Res 2005, 4(4):1442-1450.

34. Zhang F, Drabier R: SASD: the Synthetic Alternative Splicing Database for identifying novel isoform from proteomics. BMC bioinformatics 2013, , 14 Suppl 14: S13.

35. Zhang F, Drabier R: IPAD: the Integrated Pathway Analysis Database for Systematic Enrichment Analysis. BMC bioinformatics 2012, , 13 Suppl 15: S7.
36. Flicek P, Ahmed I, Amode MR, Barrell D, Beal K, Brent S, Carvalho-Silva D, Clapham P, Coates G, Fairley S, et al: Ensembl 2013. Nucleic Acids Res 2013, 41(Database):D48-55.

37. Eng JK, McCormack AL, Yates lii JR: An approach to correlate tandem mass spectral data of peptides with amino acid sequences in a protein database. Journal of the American Society for Mass Spectrometry 1994, 5(11):976-989.

38. Bjornson RD, Carriero NJ, Colangelo C, Shifman M, Cheung KH, Miller PL, Williams K: X!!Tandem, an improved method for running X!tandem in parallel on collections of commodity computers. J Proteome Res 2008, 7(1):293-299.

39. Koenig T, Menze BH, Kirchner M, Monigatti F, Parker KC, Patterson T, Steen JJ, Hamprecht FA, Steen H: Robust prediction of the MASCOT score for an improved quality assessment in mass spectrometric proteomics. $J$ Proteome Res 2008, 7(9):3708-3717.

40. Geer LY, Markey SP, Kowalak JA, Wagner L, Xu M, Maynard DM, Yang X, Shi W, Bryant SH: Open mass spectrometry search algorithm. J Proteome Res 2004, 3(5):958-964.

41. Johnson RS, Taylor JA: Searching sequence databases via de novo peptide sequencing by tandem mass spectrometry. Mol Biotechnol 2002, 22(3):301-315.

42. Ma B, Zhang K, Hendrie C, Liang C, Li M, Doherty-Kirby A, Lajoie G: PEAKS: powerful software for peptide de novo sequencing by tandem mass spectrometry. Rapid Commun Mass Spectrom 2003, 17(20):2337-2342.

43. Zhang F, Drabier R: IPAD: the Integrated Pathway Analysis Database for Systematic Enrichment Analysis. BMC Bioinformatics 2012, 13(14).

44. Wang M, Witzmann FA, Lyubimov AV: Proteomics in Drug Discovery and Development. Encyclopedia of Drug Metabolism and Interactions John Wiley \& Sons, Inc; 2011.

45. Kent WJ, Sugnet CW, Furey TS, Roskin KM, Pringle TH, Zahler AM, Haussler D: The human genome browser at UCSC. Genome Res 2002, 12(6):996-1006.

doi:10.1186/1752-0509-7-S5-S8

Cite this article as: Zhang et al.: Novel alternative splicing isoform biomarkers identification from high-throughput plasma proteomics profiling of breast cancer. BMC Systems Biology 2013 7(Suppl 5):S8.

\section{Submit your next manuscript to BioMed Central and take full advantage of:}

- Convenient online submission

- Thorough peer review

- No space constraints or color figure charges

- Immediate publication on acceptance

- Inclusion in PubMed, CAS, Scopus and Google Scholar

- Research which is freely available for redistribution 